Results Out of 653 patients, 51 had positive influenza detection and unanimous expert diagnosis: 44 simple viral infections and 7 influenza with bacterial coinfections (Figure 1). Antibiotics were prescribed to all 7 cases of influenza with bacterial coinfection and to 20/44 cases adjudicated as simple viral infections, indicating an overuse rate of $45 \%$. The assay correctly classified 40 of the 44 simple viral cases (out of the remaining 4, 2 were assigned viral with bacterial coinfection and 2 received equivocal outcomes) as well as 5 of the 7 viral with bacterial coinfection cases (the remaining 2 received equivocal outcomes) supporting the assay's potential to reduce antibiotic overuse 5 -fold (from $45 \%$ to $4 / 44=9 \%$, $\mathrm{P}<0.001$ ).

Conclusion The host-response assay can differentiate between simple influenza and influenza patients with bacterial coinfection, with potential to reduce antibiotic overuse. Utility studies are warranted to demonstrate that the assay can safely assist physicians in correct management of influenza patients

\section{OC4 THE QUALITY OF CHILD HEALTHCARE: A COMPARISON AMONG 30 EUROPEAN COUNTRIES}

${ }^{1}$ Fabrizio Pecoraro*, ${ }^{2}$ llaria Rocco, ${ }^{2}$ Barbara Corso, ${ }^{3}$ Oscar Tamburis, ${ }^{2}$ Nadia Minicuci, ${ }^{4}$ Daniela Luzi. 'A Institute for research on Population and Social Policies, National Research Council, Rome, Italy, Rome, Italy; '2Neuroscience Institute, National Research Council, Padua, Italy; ${ }^{3}$ Institute for research on Population and Social Policies, National Research Council, Rome, Italy; ${ }^{4}$ Institute for research on Population and Social Policies, National Research Council, Rome, Italy, Rome, Italy

\subsection{6/archdischild-2019-epa.4}

Background and goals Even though the conceptions and contents of quality of healthcare for children have substantially improved in the last decades, little is known about the routine adoption of measures used at national/regional level to evaluate childcare. This paper intends to investigate country practices in the evaluation of child healthcare providing insights on the priority posed in 30 European/EEA countries. It reports some of the main results achieved during the MOCHA (Models of Child Health Appraised) project.

Methods An ad hoc questionnaire was developed and sent to country-specific experts (CAs), appointed for each of the MOCHA's countries, to gather information on measures adopted to evaluate the quality of child care at country/ regional level. Results were analysed and compared on the basis of a two-level hierarchical map of domains that helped the comparison and also allowed the analysis on the breadth of child-centred evaluation of healthcare. The frequency of adoption of the whole set of measures was analyzed in order to investigate whether a similar behavior in terms of sparsity/ convergence is present across countries. Moreover, to identify whether a core set of common measures is adopted across countries, we analyzed measures reported by at least $25 \%$ of the CAs under the perspective of child-centeredness which may be also expressed by age ranges reported as variable of the measures and disease specificity.

Results Among the 27 CAs that completed the questionnaire, 23 countries reported to have a national/regional agency that assesses healthcare systems. 12 of them devote a specific part of the quality assessment to child health care, while the other countries integrate some child specific items within their health system evaluation. In total 352 measures were reported. The collected measures are not equally distributed among countries, as they range from a minimum of 6 measures in
Iceland to a maximum of 130 in the UK. Considering the analysis of core measures the most common one reported by 13 countries is the immunization rate, while a two other focal points can be identified in measures related to mortality and natality.

Conclusions The analyses confirmed a high variability across countries, both in number and type of measures adopted. Only a limited set of common measures, focused on immunization, mortality and hospitalization Only a limited set of common measures, focused on immunization, mortality and hospitalization, was identified, even if sparsely distributed among a restricted number of countries.

\section{OC5 'UP, AWAY AND OUT OF SIGHT' - HOW CHILDPROOF ARE CHILDPROOF MEDICATIONS?}

Aoife Cassidy*, Ronan Brady, Luke Keogh, Jean Donnelly. Our Lady's Children's Hospital Crumlin, Dublin, Ireland

10.1136/archdischild-2019-epa.5

Aims Despite numerous campaigns re storing medication safely away from children there are still many incidents of accidental poisoning each year. Parents often feel 'childproof' caps are $100 \%$ safe, even with inadequate storage. This study aims to assess how safe 'childproof' caps on medication are by determining if children of various ages can open these medication containers and if so, how quickly they can achieve this.

Methods Children aged up to 16 years old without severe physical or intellectual disabilities were included in this study. Data collection was obtained in the outpatient department while patients awaited their OPD appointment. The time taken for a child to open each 'childproof' medication container was recorded up to a maximum of two minutes. The age of the child and any relevant diagnosis was also recorded. Information on safe storage of medication was provided to parents.

Results Seventy-six children were included in this study to date. Ages ranged from 1 year to 16 years and were from a wide range of speciality clinics. Fourteen (18\%) children were able to open at least one container within 2 minutes (age range 4 to 16 years). All children 11 years and older were successful. All children aged 7 years and younger were unable to open the container, except one child who had been taught how to open his medications.

Twenty-five percent of females were able to open at least one medication bottle versus $14 \%$ of males, however there was a preponderance of females in the older age group. Of the children who were successful, the majority were able to open the container in less than 5 seconds. An incidental finding of this study was that in cases where the medication cap was not properly closed, most children were able to quickly open the container without difficulty.

Conclusion This study shows that the almost all children 7 years and younger were unable to open a childproof medication bottle in 2 minutes, however real-life data shows the majority of paracetamol and ibuprofen poisonings occur in children under 10 years old. Parents of young children often overestimate how safe 'childproof' medication containers are and underestimate what a child can do in a brief unsupervised period. Public awareness needs to be increased of the importance of proper closing and the safe storage of 'childproof' medications in order to minimize the risk of accidental poisoning. 